\title{
A Comparison of Commercial and Experimental Ultrafiltration Membranes via Surface Property Analysis and Fouling Tests
}

\author{
Huyen T. Dang, ${ }^{1}$ Roberto M. Narbaitz, ${ }^{1 *}$ Takeshi Matsuura ${ }^{2}$ and Kailash C. Khulbe ${ }^{2}$ \\ Department of Civil Engineering ${ }^{1}$ and Department of Chemical Engineering, ${ }^{2}$ \\ University of Ottawa, 161 Louis Pasteur St., Ottawa, Ontario K1N 6N5
}

\begin{abstract}
Surface modified polyethersulfone (PES) membranes via the addition of different hydrophiLic Surface Modifying Macromolecules (LSMMs) have been developed by Matsuura, Narbaitz and co-workers. This study compares the performance of the best PES-LSMM membrane with nine commercial ultrafiltration membranes manufactured using five different types of base polymers (polyethersulfone, polysulfone, celluloseacetate, polyacrylonitrile and cellulose) in the same range of molecular weight cutoff (MWCO) (from 40 to $120 \mathrm{kDal}$ ). All membranes were characterized by using atomic force microscopy, contact angle measurements, solute transport analysis and then evaluated through fouling tests with Ottawa River water (ORW). While PES-LSMM membranes had much lower initial flux than the commercial membranes, they had the highest total organic carbon (TOC) rejection $(80 \%)$ and the lowest flux reduction $(62 \%)$. For the commercial membranes, the range of TOC rejections and flux reductions were 62 to $80 \%$ and 68 to $80 \%$, respectively. Given their high TOC rejection and relatively high foulant deposition, fouling of the PES-LSMM membranes appears to be controlled by a surface gel.
\end{abstract}

Key words: ultrafiltration membranes, surface water, membrane surface characterization, fouling, humic substances

\section{Introduction}

Fouling, i.e., flux reduction with time, is one of the most serious concerns in the application of membrane processes. This phenomenon depends on many factors, including feed characteristics, membrane apparatus type, membrane characteristics and operational procedures. Many approaches have been examined to minimize the impact of membrane fouling. Among others, Matsuura, Narbaitz and co-workers (Pham 1995; Ho et al. 2000; Suk et al. 2002) have concentrated on developing membranes whose surfaces are modified by blending tailor-made surface active polymers into polyethersulfone (PES) solutions. During the casting process the surface active additives migrate to the membrane surface, thus creating asymmetric membranes with modified surfaces via a single manufacturing step. Initial work with hydrophobic tailor-made additives produced only marginally better performance in river water filtration (Mosqueda-Jimenez et al. 2004a,b,d). Recently, Rana et al. (2005) developed several different tailor-made hydrophiLic Surface Modifying Macromolecules (LSMMs) and Nguyen et al. (Submitted for publication) demonstrated LSMM addition had positive impacts on PES membrane performance, i.e., higher TOC removals and final fluxes than PES membranes without LSMM addition.

\footnotetext{
*Corresponding author; narbait@eng.uottawa.ca
}

The objective of this study was to compare the surface characteristics and performance of the best PESLSMM membrane (as identified by Nguyen et al., Submitted for publication) with nine commercial ultrafiltration (UF) membranes. The key features of this study were to: (i) prepare the optimum PES-LSMM membrane based on the previous work; (ii) characterize the properties of the experimental PES-LSMM and commercial UF membranes via solute transport tests, contact angle and surface roughness measurements; (iii) test the fouling characteristics of the membranes via filtration tests using highly coloured river water, and (iv) finally compare their performance to assess the effectiveness of PES-LSMM membranes. The performance was assessed in terms of long-term flux rates, flux reduction and TOC rejection.

\section{Materials and Methods}

\section{Materials}

Nine commercial flat sheet membranes prepared using five different base polymers (polyethersulfone [PES], polysulfone [PS], cellulose acetate [CA], polyacrylonitrile [PAN] and cellulose) were selected for testing. Details of these membranes are presented in Table 1.

The optimum tailor-made PES-LSMM membrane as identified by Nguyen et al. (Submitted for publication) was prepared using PES (Victrex 4100P, ICI Advanced Materials, Billingham, U.K., molecular weight 
17-19 kDal) and the LSMM-600 developed by Rana et al. (2005). The LSMM-600 was synthesized using a twostep solution polymerization method: the initial step involved the reaction of methylene bis- $p$-phenyl diisocyanate (MDI) with poly(propylene glycol) (PPG) in a common solvent of $\mathrm{N}, \mathrm{N}$-dimethylacetamide (DMAc), then it was terminated by the addition of poly(ethylene glycol) (PEG) with MW of 600 Daltons. LSMMs are surface-active polymeric additives, which are blended into the casting solution containing the base polymer PES and a solvent. During the casting, LSMMs diffuse and migrate to the surface of the membrane to a certain extent. As a result, the modified membrane surface becomes more hydrophilic. Reagent-grade N-methyl-2pyrrolidone (NMP) (Aldrich Chemical Company, Inc., Milwaukee, Wis.) was used as solvent for casting membranes. Polyethylene glycol (PEG) and polyethylene oxide (PEO) (Aldrich Chemical, Milwaukee, Wis.) were used as probe solutes for the solute transport tests in molecular weight cutoff (MWCO) determinations. The molecular weights of the PEG used were 10, 20 and $35 \mathrm{kDal}$ and for PEO the molecular weight was $100 \mathrm{kDal}$. Ultra-pure water was prepared with a Milli-Q Water System (Millipore, Bedford, Ma.).

Ottawa River water collected at the intake of the Britannia Water Treatment Plant on May 8, 2004, was used as feed water for the fouling tests. This water was selected because it is highly coloured and had a TOC of about $6 \mathrm{mg} / \mathrm{L}$, thus it should cause substantial natural organic matter (NOM) fouling of the membranes.

\section{Preparation of Membranes}

The experimental PES-LSMM ultrafiltration membrane evaluated in this study was prepared with a casting solution of 18 wt \% PES, 4.5 wt \% LSMM 600 and 77.5 wt $\%$ solvent NMP (Nguyen 2005). PES, NMP and LSMM 600 were completely mixed for $24 \mathrm{~h}$ in an incubator shaker (New Brunswick Scientific Co., Edison, N.J.), filtered by a nitrogen gas-driven filter using 47-mm white mitex filter media (Milipore Corp., Bedford, Ma.). The solution was then degassed via a vacuum desiccator

TABLE 1. Description of commercial membranes

\begin{tabular}{lll}
\hline Type of membrane & Manufacturer & Materials \\
\hline XM50 & Millipore & $\begin{array}{c}\text { Regenerated } \\
\text { cellulose } \\
\end{array}$ \\
Mil100 & Millipore & PES \\
FO100 & Millipore & Modified PS \\
FO50 & Millipore & Modified PS \\
PAN100 & GE Osmonics & PAN \\
PX150 & GE Osmonics & PES \\
PU40 & GE Osmonics & PES \\
EWH70 & GE Osmonics & PS \\
CQ40 & GE Osmonics & CA \\
\hline
\end{tabular}

(VWR, Mississauga, Ont.) for several hours. Casting of the PES-LSMM membrane was conducted according to the procedures described by Mosqueda-Jimenez et al. (2004a). After casting, the flat sheet membranes were stored in ultra-pure water until they were tested. For commercial membranes, since they were coated with glycerine as a preservative to prevent them from drying and cracking, they were soaked in water for three to six hours prior to use, according to manufacturer's recommendations. All membranes were cut into 52-mm diameter coupons for testing in the ultrafiltration system. For the measurement of the contact angles, the membranes were dried naturally by putting them between filter papers instead of using the more complicated ethanol solvent exchange method (Matsuura 1994). Also, this air-drying method was selected to guarantee a fair comparison since ethanol solvent exchange may alter the commercial membranes, which might already have been dried by other methods.

\section{Methods of Analysis}

Atomic force microscopy. Atomic force microscopy (AFM) is an advanced analysis technique that can produce three-dimensional images of solid surfaces at very high resolution (Chan 1994). The advantage of using AFM is that it can image non-conducting samples, such as polymers. In contrast to scanning electron microscopy (SEM), which requires a high vacuum both during heavy metal coating and during examination, AFM can be performed on wet ultrafiltration membranes (Fritzsche et al. 1992) so it is less destructive and more representative of "natural" conditions. The AFM studies were conducted in a tapping mode using a Nanoscope III equipped with a 1533D scanner (Digital Instruments, Gainesville, Fla.). Pieces of flat-sheet membranes were cut and fixed over magnetic disks with two-sided adhesive tape. The laser beam of the AFM was focused on a pre-selected spot of the surface prior to the engagement of the cantilever (Khayet et al. 2002). A tapping mode, AFM, in air was used to generate the AFM images. Based on the data, the roughness was obtained.

Contact angle measurement. Contact angle is considered the most convenient index of membrane hydrophilicity if measuring methods and times are carefully kept consistent (Amy et al. 2001), the surface of the solid is smooth, chemically homogenous and rigid (Chan 1994), and water is used as a testing liquid. The sessile drop method, which is based on the principle of the three-phase equilibrium that occurs at the solid/liquid/air interface, was used in this study. The dry membranes were cut into $5 \times 25 \mathrm{~mm}$ pieces and fixed onto $25 \times 75 \times$ $1 \mathrm{~mm}$ superfrost micro slides (VWR., Mississauga, Ont.) using invisible tape. Advancing contact angles were determined via a goniometer (C860 701, Sherr Tumico, Germany). Water was continuously added using a 
$100-\mu \mathrm{m}$ syringe at a very slow rate until the drop moved. The water drop volume ranged from 1.6 to $2.4 \mu \mathrm{L}$. The contact angle was measured immediately after the sudden movement of the water drop using the goniometer. The reported contact angles are the average of five or six measurements. According to the manufacturer, the accuracy of this analyzer is \pm 2 degrees.

Ultrafiltration testing protocol. The ultrafiltration testing protocol consisted of several stages: pre-compaction, pure water permeation (PWP) rate determinations, solute transport evaluations and river water fouling tests. The ultrafiltration apparatus consists of the six cells in series ultrafiltration system described by MosquedaJimenez et al. (2004c), where each cell is used to test one $52-\mathrm{mm}$ diameter flat sheet membrane coupon. The filtration test protocol followed was that described in Nguyen et al. (Submitted for publication).

Membranes were pre-compacted for one hour at $552 \mathrm{kPa}(80 \mathrm{psig})$ and room temperature. The purpose of pre-compaction is to minimize flux reduction due to membrane compaction while avoiding a significant impact on membrane pore structure. This was followed by $50 \mathrm{~h}$ of PWP monitoring, i.e., ultra-pure water filtration at $345 \mathrm{kPa}(50 \mathrm{psig})$ and room temperature. The pure water permeation rate was checked by measuring the volume of permeate collected over specific time intervals.

Solute transport. Solute transport was evaluated following the procedure described by Mosqueda-Jimenez (2004a). It is essentially a continuation of the ultrafiltration test in which the feed is changed to solutions of different known molecular weight solutes for one-hour periods. The feed solutions were solutions of PEG with molecular weights of 10,20 and $35 \mathrm{kDal}$ and PEO with molecular weight of $100 \mathrm{kDal}$. The tests were run at $345 \mathrm{kPa}(50 \mathrm{psig})$ pressure and room temperature $\left(23 \pm 0.2^{\circ} \mathrm{C}\right)$. The membrane system was flushed with ultra-pure water for one hour after each PEG/PEO solution circulation. At the end of the hour the PWP rate was re-measured to corroborate that the solute transport tests had not altered the flux (i.e., fouled the membrane). The feed and permeate samples were collected and analyzed for total organic carbon (TOC) concentrations using a UV-persulfate oxidation based TOC analyzer (Phoenix 8000, Teledyne-Tekmar, Mason, Ohio). The solute feed concentrations were $100 \mathrm{mg} / \mathrm{L}$ and the samples were diluted prior to analysis due to the concentration range measurable by the above analyzer. The MWCO, which is the molecular weight that would yield $90 \%$ solute separation, was determined based on the solute transport tests. These results were used to determine the pore distribution, pore density and surface porosity of each membrane following the approach of Singh et al. (1998).
Fouling test. After the solute transport analysis, the fouling test was conducted using the same experimental setup and switching the feed to Ottawa River water (ORW). ORW was used as a feed because of its relatively high colour and NOM content, and this makes it a good challenge water for the fouling tests. The system was operated at room temperature and the pressure was controlled manually using two valves and pressure gauges (MIX3, France). The 144-h test duration allowed the permeate flux to reach a pseudo-steady state. The permeate flux was determined by weighing the mass of the permeate collected during predetermined times or measuring the volume of permeate with higher permeability membranes. The feed flow was set at $1.1 \mathrm{Lpm}$ to allow the operation of two systems (six cells per each) in series with a pressure drop of less than $14 \mathrm{kPa}$ (2 psig) while creating turbulent flow conditions so as to avoid concentration polarization (Mosqueda-Jimenez 2003). The NOM rejection was quantified using the feed and permeate total organic carbon (TOC) concentrations. At the end of the fouling test, the diameter of the fouled membrane surface area was measured to permit accurate calculation of the fluxes. In addition, the mass of foulant was quantified by the technique suggested by Hong and Elimelech (1997). The NOM-fouled membranes were immersed in $25 \mathrm{~mL}$ of $0.1 \mathrm{M} \mathrm{NaOH}$ within pre-weighed beakers. After the NOM was completely removed from the membrane coupons, all the coupons were removed from the beakers. The beakers were placed in an aircirculating oven at $105^{\circ} \mathrm{C}$ for $24 \mathrm{~h}$ to evaporate the water and then reweighed. The mass of NOM calculated by the difference between the dry weight of the beakers, containers containing $\mathrm{NOM}$ and $\mathrm{NaOH}$ and that of the controls (containing just $\mathrm{NaOH}$ ).

\section{Results and Discussion}

\section{Ultrafiltration Membrane Performance and Comparison}

Pure Water Permeation and Membrane Resistance. The intrinsic membrane resistance, determined using pure water as a feed, is not only useful for modelling purposes, but also for evaluating the stability of the membrane (Cheryan 1986). This resistance, $\mathrm{R}_{\mathrm{m}}$ (or hydraulic resistance of clean membranes $\mathrm{m}^{-1}$ ), can be expressed as:

$$
\mathrm{R}_{\mathrm{m}}=\frac{\Delta \mathrm{P}_{\mathrm{T}}}{\mathrm{J} \mu}
$$

where $\Delta \mathrm{P}_{\mathrm{T}}$ is the transmembrane pressure $\left(\mathrm{N} / \mathrm{m}^{2}\right), \mathrm{J}$ is the pure water permeation flux $\left(\mathrm{m}^{3} / \mathrm{m}^{2} \mathrm{~s}\right)$ and $\mu$ is the viscosity of water $\left(\mathrm{Ns} / \mathrm{m}^{2}\right)$.

This value was evaluated after 1 -h precompaction and 50 -h permeation using ultra-pure water. From Fig. 1, it was obvious that the resistance was similar for all membranes, ranging from 9 to $13 \mathrm{~m}^{-1}$. The experi- 


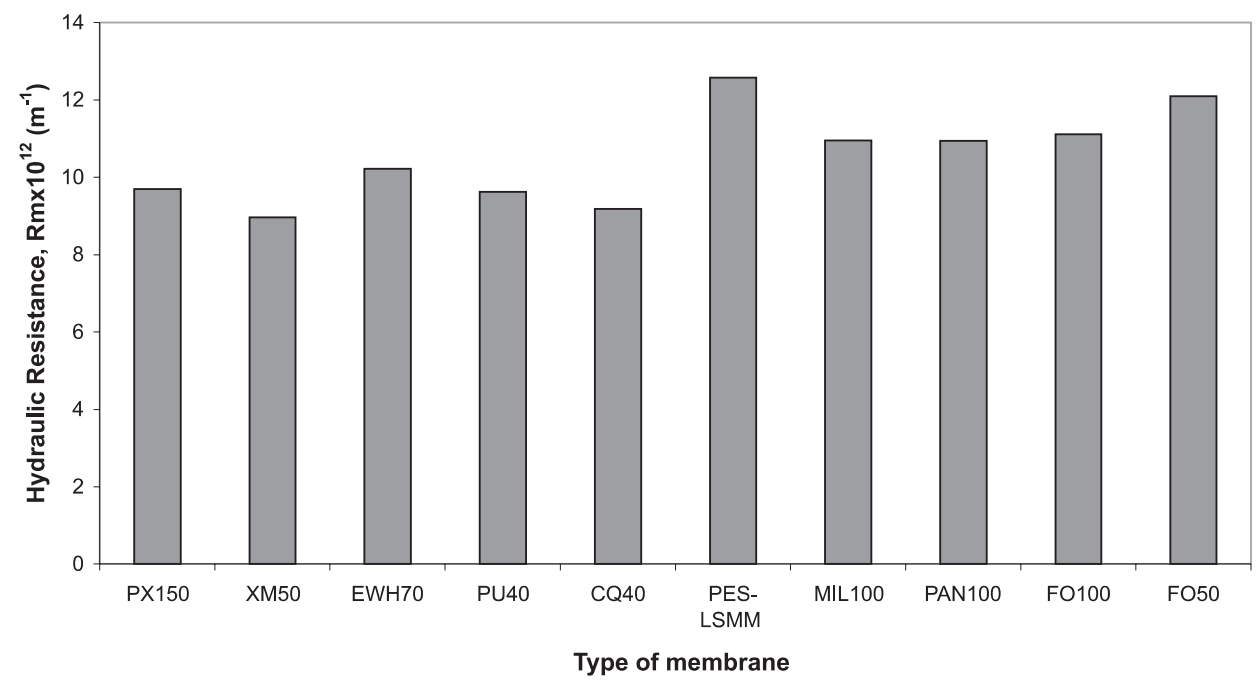

Fig. 1. Membrane hydraulic resistance of the various membranes (after PWP monitoring).

mental membrane, PES-LSMM, had the highest hydraulic resistance. The hydraulic resistance is inversely proportional to the flux, hence, the permeation rate after the 50-h test of PES-LSMM membrane was not as high as the commercial membranes. Cellulose membranes had the lowest resistance or highest permeation rate. This can be explained based on the differences in polymer structure. Cellulose membranes have a continuous sponge-like structure that makes them less susceptible to compaction and obtains higher flux while PES membranes have a structure with macrovoids below the thin separation layer (Persson et al. 1995).

Long-term fouling test. Figure 2 illustrates the flux over the various stages of the ultrafiltration protocol: precompaction, PWP, solute transport (only shown as the vertical line) and ORW fouling test. Although commercial membranes such as FO50, MIL100 or XM50 had high permeation rates at the start-up, their fluxes quickly dropped in the first six hours and gradually reached a pseudo-steady state after $80 \mathrm{~h}$. The flux decline at the beginning of the filtration test might be due to the characteristics of the pore structures of the membranes which allowed large amounts of humic substances to pass through and thus were more vulnerable to pore blocking (Nguyen 2005). The flux of the PES-LSMM membrane did not decline significantly, in particular it was held rather steady during the ORW filtration test.

Flux reduction, which is an indicator of fouling, was calculated as below:

$$
\text { Flux reduction }_{(\mathrm{t})}(\%)=100\left(1-\frac{P R_{O R W}}{P W P}\right)
$$

where PRORW is the permeate flux after the 6-d fouling test with Ottawa River water $\left(\mathrm{L} / \mathrm{m}^{2} / \mathrm{h}\right)$ and PWP is the stable pure water permeation rate $\left(\mathrm{L} / \mathrm{m}^{2} / \mathrm{h}\right)$.

The flux reductions during the six-day ORW filtration test are presented in Fig. 3. The initial fluxes of com-

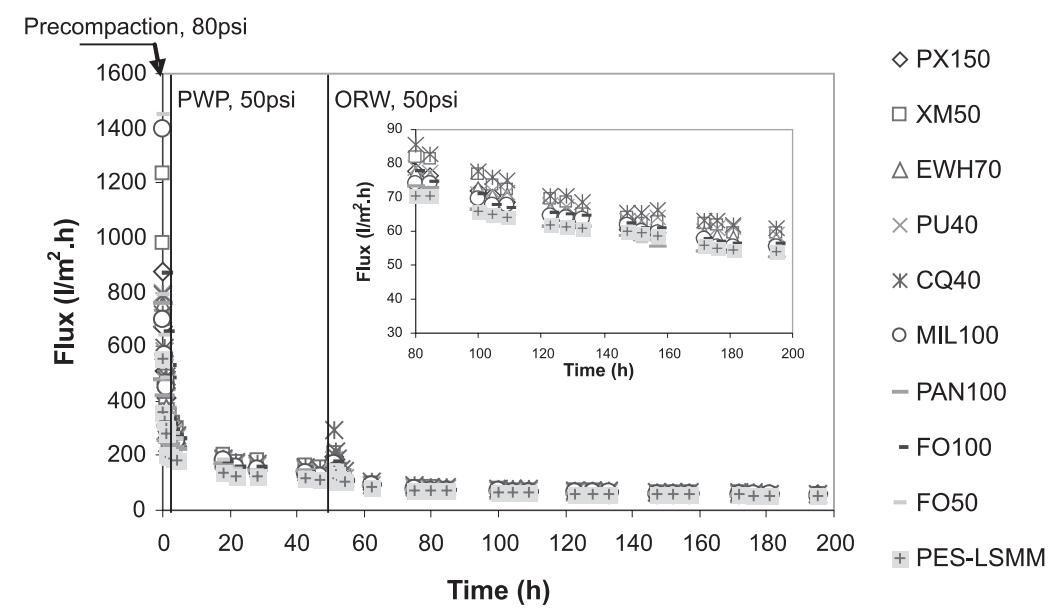

Fig. 2. Filtration flux versus time. 


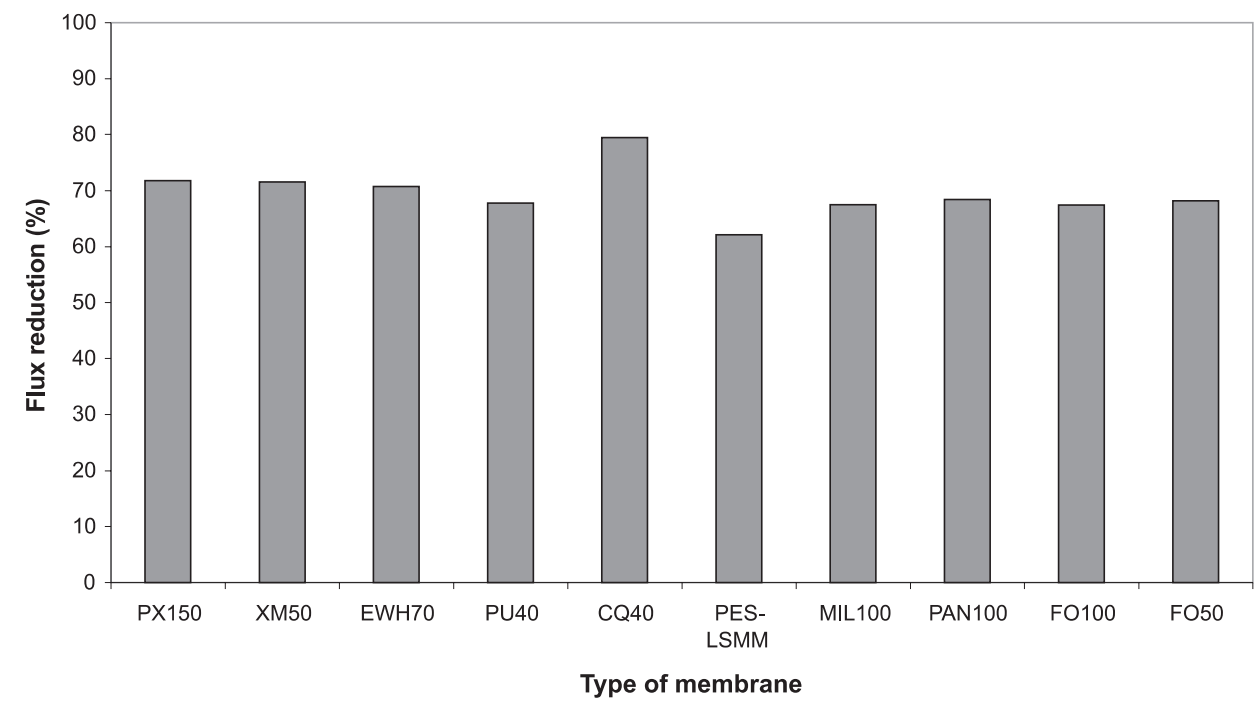

Fig. 3. Flux reduction after filtration test (144 h).

mercial membranes were relatively high compared to the modified membrane but the final fluxes showed almost similar (from $52.10 \mathrm{~L} / \mathrm{m}^{2} \mathrm{~h}$ to $60.68 \mathrm{~L} / \mathrm{m}^{2} \mathrm{~h}$ ) after the long-term filtration test. Therefore, the flux reduction of the modified membrane was lower since its initial flux was not competitive. One interesting conclusion drawn from the above results was that the flux stability of the PES-LSMM membrane was better than others. The lower flux reduction also implied it was less susceptible to fouling. This is probably due to the rather small values of mean pore sizes, which are discussed in a later section.

Humic substances deposition and TOC rejection. Removal of NOM, or more specifically humic substances, is important in water treatment primarily because they react with chlorine to form harmful disinfection by-products (DBPs). In membrane technology, the amount of humic substances deposited on membrane surfaces was studied since it is related to the cleaning frequency. This deposition layer is known as the fouling layer. The more quickly the fouling layer grows, the greater the frequency of cleaning required. The amount of chemicals used for cleaning the membranes with highly accumulated NOM is typically greater than those having less NOM deposition.

It is also important to quantify TOC rejection, which was calculated via the following equation:

$$
\text { TOC rejection }(\%)=100 \times\left(1-\frac{T^{O O C_{p}}}{T_{O C C_{f}}}\right)
$$

where $\mathrm{TOC}_{\mathrm{f}}$ and $\mathrm{TOC}_{\mathrm{p}}$ are total organic carbon concentration $(\mathrm{mg} / \mathrm{L})$ in the feed and permeate, respectively.

Small amounts of NOM deposition and high TOC rejection suggests that there is more internal fouling, i.e., more humic substances penetrate into the pores and plug them. Internal fouling is generally irreversible and requires more expensive and complex cleaning procedures. High amounts of NOM deposition accompanied by high TOC removals, on the other hand, indicate that surface fouling is dominant. This fouling layer is generally reversible and can be easily controlled by high turbulence, regular cleaning and using hydrophilic or charged membranes to minimize adhesion to the membrane surface (Baker 2004). Ideally, the membranes that foul predominantly on the surface are preferred since they are easier to clean and maintain. Figure 4 shows that the experimental PES-LSMM membrane has the highest TOC rejection and relatively high NOM deposition $\left(9.03 \times 10^{-3} \mathrm{~g}\right)$. Thus, it is hypothesized that for the apparatus, test conditions and water tested, the modified PES-LSMM membranes' dominant fouling mechanism was surface fouling. The percentage of TOC removal of the experimental membrane was significant. Its initial TOC rejection $(72 \%)$ was the highest and increased to $80 \%$ by the end of the six-day filtration test. This value was higher than those reported in the literature (Nguyen et al., Submitted for publication; Rana et al. 2005) for membranes prepared in a similar fashion. The initial TOC rejections of the commercial membranes ranged from 34 to $61 \%$, while the long-term rejections of these membranes ranged from 64 to $70 \%$. These above values are considerably higher than the values of 20 to $40 \%$ reported in other studies for commercial UF membranes (Mosqueda-Jimenez 2003). This may be attributed to the characteristics of the fouling test apparatus, the characteristics of the test water (which in this case was a raw river water), the duration of the fouling test, etc. To understand further the impact of surface gel, the percentages of initial and final TOC rejection were assessed in this study (Fig. 5). The initial TOC rejection usually reflects the rejection of the membrane without the impact of the fouling layer. Gradually, the NOM deposi- 


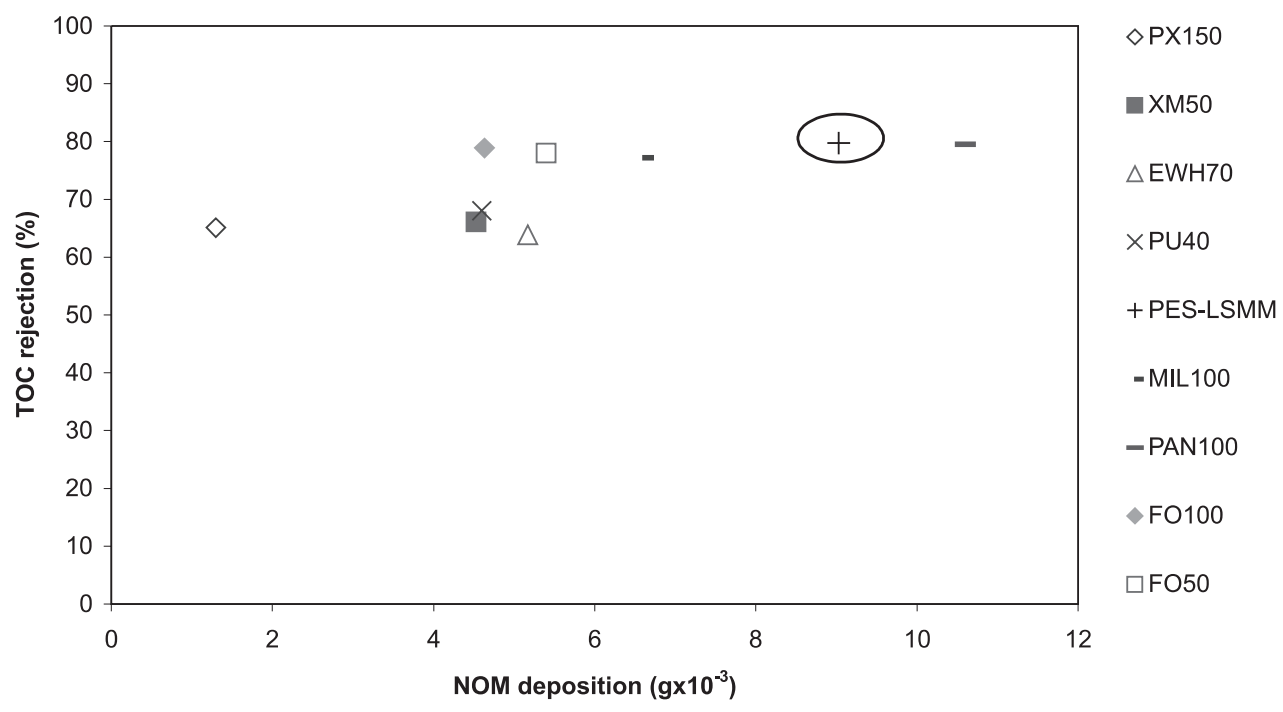

Fig. 4. Final TOC rejection versus NOM deposition.

tion layer increases and results in the increasing selectivity of the membranes. The final TOC rejection, therefore, can be higher than the initial one. Comparing the maximum values in the initial and the final rejections of the PES-LSMM membrane, the difference was not very significant (from over $70 \%$ to approximately $80 \%$ ). It proved that for the experimental membrane, TOC rejection by the membrane's surface gel was not so significant. Evaluation of the membrane retention would be possibly reliable based on the initial percentage of rejection since the fouling layer may be controlling in many cases but not all cases.

\section{Correlation between Surface Properties and Fouling Phenomenon}

Hydrophilicity. Advancing contact angles of all membranes were less than $53^{\circ}$ (see Table 2) demonstrating that they are all hydrophilic membranes. The tailor- made membrane with a contact angle of $52.5^{\circ}$ was the least hydrophilic one. This value of contact angle was approximately $20^{\circ}$ smaller than that reported by Nguyen et al. (Submitted for publication). This could be attributed to the membrane drying method. In this study, the membranes were dried naturally by placing them between filter papers while the membranes developed by Nguyen (2005) were dried with traditional ethanol solution. The impacts of different drying methods require further study. In addition, Nguyen (2005) used a more sophisticated goniometer which may have contributed to the observed differences to some extent.

Upon completing the test with Ottawa River water, the fouled membranes were submerged in $0.1 \mathrm{M} \mathrm{NaOH}$ solution for one hour for cleaning, and then rinsed by immerging in ultra-pure (MQ) water for $48 \mathrm{~h}$ prior to being dried naturally. The contact angles of these membranes were measured after drying them for five days and compared with the contact angles of clean membranes.
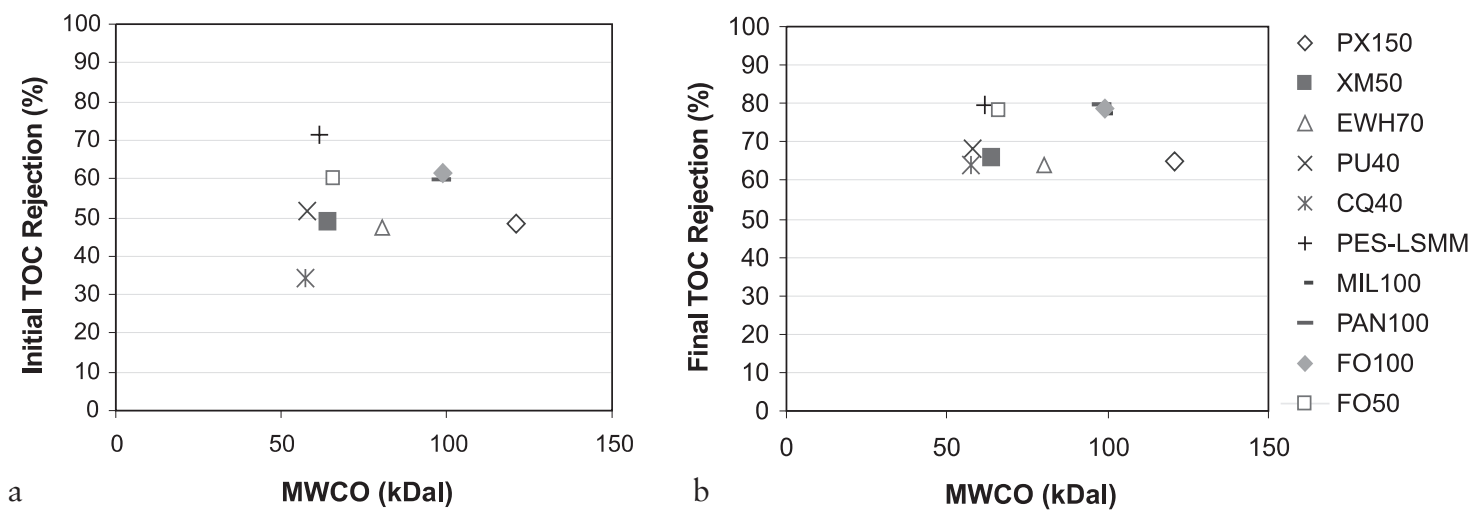

Fig. 5. Impact of surface gel through: (a) initial TOC rejection and (b) final TOC rejection (after the fouling test with Ottawa River water). 
TABLE 2. Contact angle measurements before and after the fouling test

\begin{tabular}{lccccc}
\hline & \multicolumn{2}{c}{$\begin{array}{c}\text { Before the fouling test } \\
\text { Membranes }\end{array}$} & & & \multicolumn{2}{c}{ After the fouling test } \\
\cline { 2 - 3 } & $\begin{array}{c}\text { Mean contact angle } \\
\left(^{\circ}\right)\end{array}$ & $\begin{array}{c}\text { Standard } \\
\text { deviation }\end{array}$ & & $\begin{array}{c}\text { Mean contact angle } \\
\left({ }^{\circ}\right)\end{array}$ & $\begin{array}{c}\text { Standard } \\
\text { deviation }\end{array}$ \\
\hline PX150 & 44.22 & 0.62 & & 46.58 & 2.18 \\
XM50 & 16.50 & 2.65 & & 44.70 & 3.04 \\
EWH70 & 48.30 & 2.73 & & 35.97 & 1.63 \\
PU40 & 36.00 & 1.95 & & 51.80 & 3.62 \\
CQ40 & 29.36 & 2.72 & & 40.17 & 0.29 \\
PES-LSMM & 52.47 & 1.17 & & 50.97 & 1.90 \\
MIL100 & 47.67 & 1.21 & & 48.95 & 1.28 \\
PAN100 & 34.53 & 0.41 & & 35.08 & 1.83 \\
FO100 & 51.00 & 2.02 & & 46.72 & 2.03 \\
FO50 & 50.28 & 1.24 & & 46.50 & 3.91 \\
\hline
\end{tabular}

The hydrophobicity of almost all membranes was found to either increase or decrease after the filtration test. This maybe depends on the NOM characteristics and membrane types. No satisfactory explanation of this phenomenon was given in the literature. More sophisticated tests are required to have a better understanding of these seemingly inconsistent results. However, it is worth noting that the contact angles of all membranes after fouling became more uniform. The difference between the lowest and the highest membrane contact angles decreased from 36 to $16.7^{\circ}$ due to the filtration test. This suggests that some foulants penetrated into and stayed in the membrane pores without being removed by $\mathrm{NaOH}$ cleaning.

Pore characteristics. The pores of all tested membranes were characterized by the solute transport method and the results are summarized in Table 3. Efficient membranes should have small pore sizes, high pore density and high surface porosity (Alsari et al. 2001) so that they can remove more contaminants such as humic substances from water, and yet achieve high permeation fluxes. Based on the data in Table 3, CQ40 and PU40 seemed to be the best that satisfied these requirements, followed by XM50 and PES-LSMM membranes.
Figure 6 presents the correlation between pore characteristics and molecular weight cutoff (MWCO). There was a clear trend that as MWCO increased, the mean pore size increased. It completely follows the logical concept of membrane technology since MWCO is defined as the molecular weight that yields $90 \%$ solute separation and smaller MWCO values are only obtained for membranes having smaller pore sizes. MWCO of the commercial membranes determined by solute transport method in this study differed somewhat from the estimated values provided by the suppliers. The reasons may be due to different characterization methods and different histories of membranes (i.e., length of the storage period prior to use). Cho et al. (2002) also reported that an effective MWCO is not usually the same as a nominal MWCO provided by the manufacturer. From Fig. 6, the pore density significantly decreased with the increasing MWCO. It may be explained by the fact that to yield similar fluxes, membranes with smaller pores (smaller MWCO) often have higher pore densities. The tailor-made membranes, which had MWCO of approximately $62 \mathrm{kDal}$, had a low MWCO, small mean pore size and high pore density. The fractionation study of Ottawa River water (ORW) showed that $99 \%$ of NOM had molecular weights less

TABLE 3. Pore characteristics and MWCO of tested membranes ${ }^{\mathrm{a}}$

\begin{tabular}{lccccc}
\hline Membranes & $\begin{array}{c}\text { Nominal MWCO } \\
(k D a l)\end{array}$ & $\begin{array}{c}\text { Measured MWCO } \\
(k D a l)\end{array}$ & $\begin{array}{c}\text { Pore density } \\
\left(\text { No. of pores } / m^{2}\right)\end{array}$ & $\begin{array}{c}\text { Surface porosity } \\
(\%)\end{array}$ & $\begin{array}{c}\text { Mean pore size } \\
(\mathrm{nm})\end{array}$ \\
\hline PX150 & 150 & 120.87 & 4.52 & 0.0008 & 6.83 \\
XM50 & 50 & 64.13 & 15.14 & 0.0017 & 5.43 \\
EWH70 & 70 & 80.39 & 9.41 & 0.0012 & 5.90 \\
PU40 & 40 & 58.06 & 19.90 & 0.0018 & 4.80 \\
CQ40 & 40 & 57.59 & 19.55 & 0.0019 & 5.14 \\
PES-LSMM & 60 & 61.64 & 15.79 & 0.0016 & 5.05 \\
MIL100 & 100 & 98.52 & 6.63 & 0.0010 & 5.19 \\
PAN100 & 100 & 98.21 & 7.52 & 0.0010 & 5.40 \\
FO100 & 100 & 98.91 & 13.42 & 0.0014 & 5.12 \\
FO50 & 50 & 66.01 & &
\end{tabular}

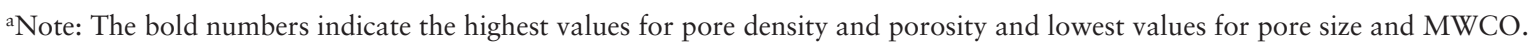




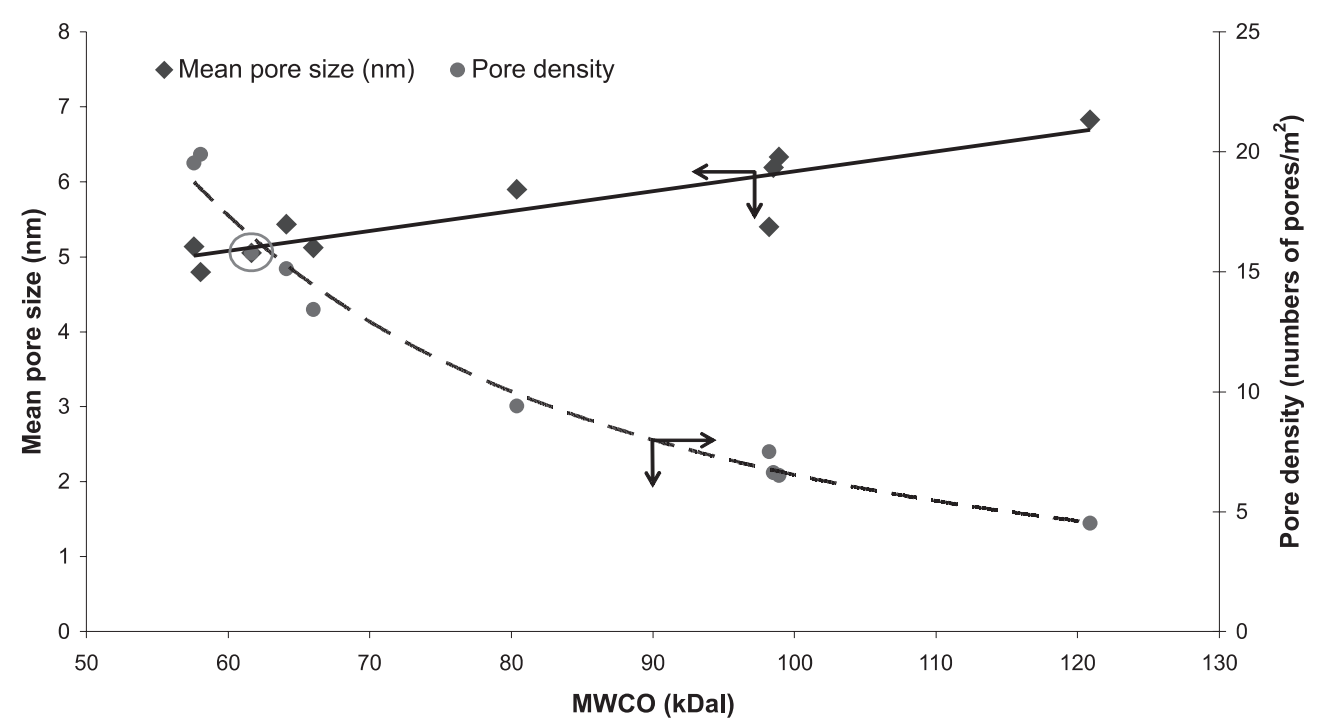

Fig. 6. Correlation of MWCO and pore characteristics.

than $30 \mathrm{kDa}$. On the other hand, the maximum NOM rejection of about $80 \%$ was achieved with the membranes with MWCO above $50 \mathrm{kDal}$. Hence, it is proved that the effective MWCO of the membranes was much lower than the MWCOs measured in this work or those supplied by the manufacturers. The effects of electrostatic repulsion and hydrodynamic operating conditions are potential reasons for this discrepancy (Cho et al. 2000). MWCO is therefore not a precise indicator for membrane performance evaluation.

Roughness. Surface morphology may be an important factor affecting the performance of membranes. Membrane morphology can be examined through AFM measurements such as the mean roughness, $\mathrm{Ra}$, which represents the mean value of surface relative to the centre plane for which the volume enclosed by the images above and below this plane are equal. The three-dimensional images of all membrane surfaces showed that most membranes were not as smooth as cellulose acetate (CQ40) and polyethersulfone (PU40) membranes. The PESLSMM membrane had a rather high roughness value.

Surface roughness is usually related to many other parameters. Singh et al. (1998) found that roughness became higher when MWCO increased since high MWCO had less tightly packed nodule aggregates in the skin layer. The same trend was observed by many other researchers (Fritzsche et al. 1992; Bessieres et al. 1996). It was confirmed again in this study that PU40 and XM40 membranes, which had lowest values of MWCO, showed the smoothest surfaces. Hirose et al. (1996) found that an increase in surface roughness resulted in a higher water permeation flux. For PVDF membranes, Khayet et al. (2004) observed that the measured pure water flux increased with the increase of roughness and the roughness parameter was higher for membranes hav- ing larger pore sizes and nodule sizes as well as higher surface porosity.

Figure 7 shows that roughness increased with an increase in the contact angles except for XM50. The water permeation rate will increase with a decrease in the contact angle, which means an increase in hydrophilicity. In fact, both cellulose (XM50) and cellulose acetate (CQ40) membranes that correspond to the lowest contact angles showed the highest PWP values (see Fig. 1 where the hydraulic resistance of the membranes is given). Then, from the trend shown in Fig. 7, it is concluded that the smoother membranes will have higher values of PWP. This seems contradictory to the results reported by Khayet et al. (2004) and Hirose et al. (1996). It should, however, be noted that the results presented in Fig. 7 involve membranes made of many different materials. On the other hand the data shown by Khayet et al. (2004) and Hirose et al. (1996) were obtained from membranes prepared from the same base polymeric material. It should also be emphasized that cellulose and cellulose acetate membranes were the smoothest and the most hydrophilic among the tested membranes.

The impact of roughness on foulant deposition is also a topic of interest. Assuming no other factors are at play, membranes that have higher roughness should retain more foulants since it is easier for them to accumulate in the valleys within the rough membrane surfaces. Roughness can impact the interactions between particles and membrane surface resulting in enhanced attachment of particles onto the surface and hence more severe fouling (Elimelech et al. 1997). Figure 8 shows the correlation of roughness and fouling phenomenon in terms of flux reduction. Statistical analysis showed that no clear trend could be found with increasing roughness for the conditions tested. Again, the reason might be attributed to different base polymer membranes. Gener- 


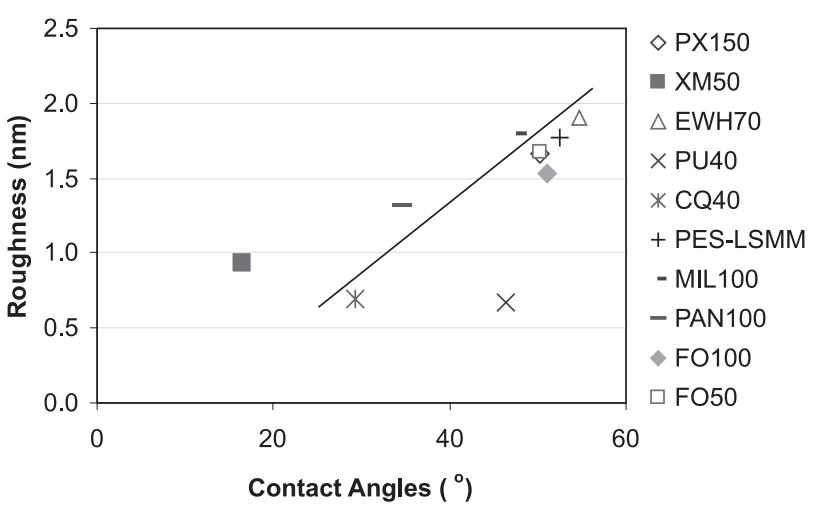

Fig. 7. Relation of hydrophilicity and roughness.

ally, membranes prepared of the same base polymer membranes with smoother surfaces should have less fouling, and thus less flux reduction.

\section{Conclusions}

The characteristics and performance of nine commercial ultrafiltration membranes were compared with those of an experimental membrane. The PES-LSMM experimental membrane had the highest flux resistance due to its low initial PWP flux and comparable long-term fluxes to those of the other commercial membranes. Based on the pore characterization and roughness measurement, the modified cellulose acetate (CQ40) and polyethersulfone (PU40) membranes appeared to be the most promising in terms of fluxes and fouling potentials. Considering the MWCO of membranes, molecular weight distribution of NOM and NOM rejection, it is noted that MWCO was proved not a truly precise indicator for the prediction of NOM rejection. The experiments showed that the membrane with the highest NOM rejection (PES-LSMM) was not the one having the lowest MWCO. The highest TOC rejection (approximately 80\%) was achieved by the tight, tailor-made PES-LSMM membrane. This TOC rejection was relatively high compared with the 20 to $40 \%$ reported in the literature. Thus, LSMM was proved to have a positive impact on membrane modification. Future research on fabrication of LSMM hollow fiber UF membranes as well as the impact of cleaning on their performance should be implemented to obtain the full understanding of the effects of these surface active molecules.

\section{Acknowledgements}

We gratefully acknowledge the financial support from the Vietnam Government (Vietnamese Overseas Scholarship Programs-VOSP) and Materials and Manufacturing Ontario (MMO) for this research. Sincere thanks to Dipak Rana of the Industrial Membrane Research Institute, Department of Chemical Engineering, University of Ottawa, for his cooperation in the LSMM synthesis.

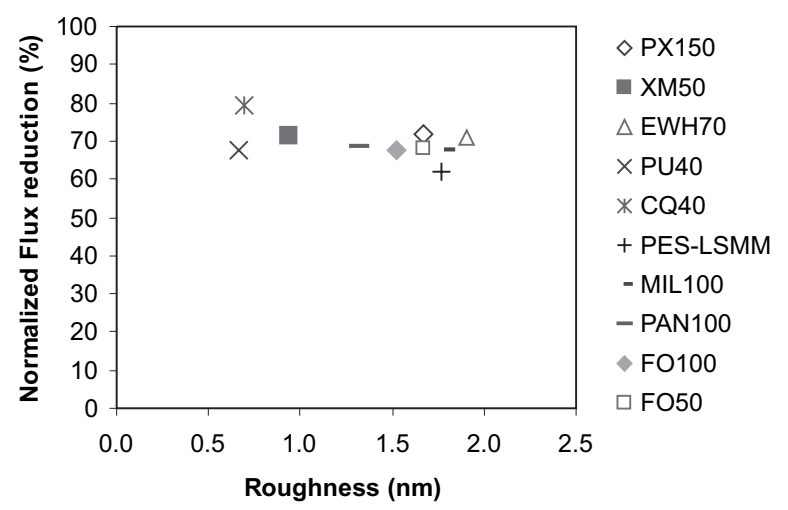

Fig. 8. Impact of surface roughness on membrane fouling.

\section{References}

Alsari AM, Khulbe KC, Matsuura T. 2001. The effect of sodium dodecyl sulfate solutions as gelation media on the formation of PES membranes. J. Membr. Sci. 188:279-293.

Amy G, Clark M, Pellegrino J. 2001. NOM rejection by, and fouling of, NF and UF membranes. Project \#390, AWWA Research Foundation, Denver, Colo.

Baker RW. 2004. Membrane technology and applications. John Wiley \& Sons, Ltd. Hoboken, N.J.

Bessieres A, Meireles M, Coratger R, Beauvillain J, Sanchez V. 1996. Investigations of surface properties of polymer membranes by near field microscopy. J. Membr. Sci. 109:271-284.

Chan CM. 1994. Polymer surface modification and characterization. Hanser Publishers, New York.

Cheryan M. 1986. Ultrafiltration handbook. Technomic Publishing Co., Lancaster, Pa.

Cho J, Amy G, Pellegrino J. 2000. Membrane filtration of natural organic matter: comparison of flux decline, NOM rejection, and foulants during filtration with three UF membranes. Desalination 127:283-298.

Cho J, Sohn J, Choi H, Kim IS, Amy G. 2002. Effects of molecular weight cutoff, $\mathrm{f} / \mathrm{k}$ ratio (a hydrodynamic condition), and hydrophobic interactions on natural organic matter rejection and fouling in membranes. J. Water Supp. Res. Technol. AQUA 51(2):109-123.

Elimelech M, Xiaohua Z, Childress AE, Seungkwan H. 1997. Role of membrane surface morphology in colloidal fouling of cellulose acetate and composite aromatic polyamide reverse osmosis membranes. J. Membr. Sci. 127:101-109.

Fritzsche K, Arevalo AR, Connolly AF, Moore MD, Elings V, Wu CM. 1992. The structure and morphology of the skin of polyethersulfone ultrafiltration membranes: a comparative atomic force microscope and scanning electron microscope study. J. Appl. Polym. Sci. 45:1945-1956.

Hirose M, Itoh H, Minamizaki Y, Kamiyama Y. 1996. Proceedings of 1996 International Congress on Membranes and Membrane Processes, Yokohama, p. 178-179. 
Ho JYC, Matsuura T, Santerre JP. 2000. The effect of fluorinated surface modifying macromolecules on polyethersulfone membranes. J. Biomater. Sci. Polym. 11:1085-1104.

Hong S, Elimelech M. 1997. Chemical and physical aspects of natural organic matter (NOM) fouling of nanofiltration membranes. J. Membr. Sci. 132:159-181.

Khayet M, Khulbe KC, Matsuura T. 2004. Characterization of membranes for membrane distillation by atomic force microscopy and estimation of their water vapor transfer coefficients in vacuum membrane distillation process. J. Membr. Sci. 238:199-211.

Khayet M, Suk DE, Narbaitz RM, Santerre JP, Matsuura T. 2002. Study on surface modification by surfacemodifying macromolecules and its application in membrane separation processes. J. Appl. Polym. Sci. 89: 2902-2916.

Matsuura T. 1994. Synthetic membranes and membrane separation processes. CRC Press, Boca Raton, Fla.

Mosqueda-Jimenez DB. 2003. Impact of manufacturing conditions of polyethersulfone membranes on final characteristics and fouling reduction. Ph.D. thesis, University of Ottawa, Ottawa, Ont.

Mosqueda-Jimenez DB, Narbaitz RM, Matsuura T. 2004a. Impact of the membrane surface modification on the treatment of surface water. J. Environ. Eng. 130: 1450-1459.

Mosqueda-Jimenez DB, Narbaitz RM, Matsuura T. 2004b. Manufacturing conditions of surface-modified membranes: effects on ultrafiltration performance. Sep. Puri. Technol. 37:51-67.

Mosqueda-Jimenez DB, Narbaitz RM, Matsuura T. 2004c. Membrane fouling test: apparatus evaluation. J. Environ. Eng. 130:90-99.

Mosqueda-Jimenez DB, Narbaitz RM, Matsuura T, Chowdhury G, Pleizier G, Santerre JP. 2004d. Influence of processing conditions on the properties of ultrafiltration membranes. J. Membr. Sci. 231:209-224.

Nguyen HA. 2005. Membrane fouling reduction by the incorporation of hydrophilic surface modifying macromolecules in ultrafiltration membrane manufacturing. M.A.Sc thesis, University of Ottawa, Ottawa, Ont.

Nguyen HA, Narbaitz RM, Matsuura T. Submitted for publication.

Persson KM, Gekas V, Trägårdh G. 1995. Study of membrane compaction and its influence on ultrafiltration water permeability. J. Membr. Sci. 100:155-162.

Pham VA. 1995. Surface modifying macromolecules for enhancement of polyethersulfone pervaporation mem- brane performance. M.A.Sc. thesis, University of Ottawa, Ottawa, Ont.

Rana D, Matsuura T, Narbaitz RM, Feng C. 2005. Development and characterization of novel hydrophilic surface modifying macromolecule for polymeric membranes. J. Membr. Sci. 249:103-112.

Singh S, Khulbe KC, Matsuura T, Ramamurthy P. 1998. Membrane characterization by solute transport and atomic force microscopy. J. Membr. Sci. 142:111-127.

Suk DE, Chowdhury G, Matsuura T, Narbaitz RM, Santerre P, Pleizier G, Deslandes Y. 2002. Study on the kinetics of surface migration of surface modifying macromolecules in membrane preparation. Macromolecules 35:3017-3021.

Received: November 15, 2005; accepted: February 13, 2006.

\section{List of Notations and Abbreviations}

\begin{tabular}{|c|c|}
\hline $\mathrm{R}_{\mathrm{m}}$ & Membrane resistance $\left(\mathrm{m}^{-1}\right)$ \\
\hline $\mathrm{R}_{\mathrm{a}}$ & Mean roughness $(\mathrm{nm})$ \\
\hline$\Delta \mathrm{P}_{\mathrm{T}}$ & Transmembrane pressure $\left(\mathrm{N} / \mathrm{m}^{2}\right)$ \\
\hline $\mathrm{J}$ & Pure water permeation flux $\left(\mathrm{m}^{3} / \mathrm{m}^{2} \mathrm{~s}\right)$ \\
\hline$\mu$ & Viscosity $\left(\mathrm{Ns} / \mathrm{m}^{2}\right)$ \\
\hline PRORW & Permeate flux after fouling test $\left(\mathrm{L} / \mathrm{m}^{2} \mathrm{~h}\right)$ \\
\hline PWP & Pure water permeation $\left(\mathrm{L} / \mathrm{m}^{2} \mathrm{~h}\right)$ \\
\hline $\mathrm{TOC}_{\mathrm{f}}$ & Total organic carbon in the feed $(\mathrm{mg} / \mathrm{L})$ \\
\hline $\mathrm{TOC}_{\mathrm{p}}$ & Total organic carbon in the permeate $(\mathrm{mg} / \mathrm{L})$ \\
\hline $\mathrm{AFM}$ & Atomic force microscopy \\
\hline $\mathrm{C}$ & Cellulose \\
\hline CA & Cellulose acetate \\
\hline DMAc & N,N-dimethylacetamide \\
\hline LSMMs & $\begin{array}{l}\text { HydrophiLic Surface Modifying } \\
\text { Macromolecules }\end{array}$ \\
\hline MDI & Methylene bis- $p$-phenyl diisocyanate \\
\hline MWCO & Molecular weight cutoff (kDal) \\
\hline NMP & N-methyl pyrrolidone \\
\hline NOM & Natural organic matter \\
\hline ORW & Ottawa River water \\
\hline PAN & Polyacrylonitrile \\
\hline PEG & Polyethylene glycol \\
\hline $\mathrm{PEO}$ & Polyethylene oxide \\
\hline PES & Polyethersulfone \\
\hline PPG & Poly(propylene glycol) \\
\hline PS & Polysulfone \\
\hline PVDF & Poly(vinylidene-fluoroethylene) \\
\hline UF & Ultrafiltration \\
\hline
\end{tabular}

